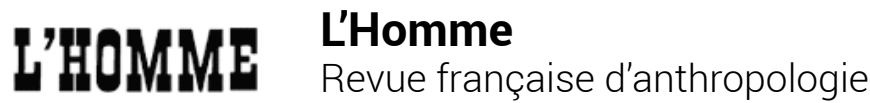

166 | avril-juin 2003

Malinowski, Faulkner. Culture et cognition. Souvenir et héritage

\section{La Russie, le temps et l'espace}

Transformations du socio-cosmisme et construction d'une modernité hybride

\section{Stéphane Vibert}

\section{CpenEdition}

\section{Journals}

Édition électronique

URL : http://journals.openedition.org/lhomme/221

DOI : 10.4000//homme.221

ISSN : 1953-8103

Éditeur

Éditions de l'EHESS

Édition imprimée

Date de publication : 30 juin 2003

Pagination : 145-170

ISBN : 2-7132-1805-5

ISSN : 0439-4216

Référence électronique

Stéphane Vibert, «La Russie, le temps et l'espace », L'Homme [En ligne], 166 | avril-juin 2003, mis en ligne le 08 septembre 2008, consulté le 20 avril 2019. URL : http://journals.openedition.org/ Ihomme/221 ; DOI : 10.4000//homme.221 


\title{
La Russie, le temps et l'espace
}

\author{
Transformations du socio-cosmisme \\ et construction d'une modernité hybride
}

Stéphane Vibert

L'

UNE DES SPÉCIFICITÉS socio-historiques de la Russie est d'avoir de tout temps posé son appartenance à l'Europe comme donnée problématique de son existence en tant qu'unité nationale. À partir du XVI ${ }^{\mathrm{e}}$ siècle, la Russie s'est pensée à la fois comme partie intégrante de la civilisation européenne (notamment par ses alliances matrimoniales princières) et comme "autre» d'un Occident souvent idéalisé, parfois diabolisé, construit pour ainsi dire "en creux " à travers ses valeurs jugées consubstantielles de liberté individuelle, de rationalisme et de développement scientifique et technique. Un Occident également toujours perçu par la pensée russe en termes d'espace géographique et de localisation temporelle particuliers, axiologiquement marqués (Lotman-Ouspenski 1990 : 52). L'étude de la "hiérarchie de valeurs" russe (au sens défini par Louis Dumont 1983), selon une dynamique historique perceptible au cours des périodes «tsariste " (d'Ivan III à Pierre le Grand, XVI ${ }^{\mathrm{e}}$-XVIII ${ }^{\mathrm{e}}$ siècles), puis "impériale " (inaugurée par Pierre) permet ainsi d'appréhender non seulement l'acculturation de la variante nationale russe au sein de «l'idéologie moderne ", mais également d'enrichir le contenu même de cette configuration moderne qui, loin d'être partout uniforme et homogène, utilise les éléments holistes autochtones afin de conforter sa puissance d'imprégnation et d'approfondir son adaptabilité.

"La société est elle-même institution d'une temporalité "implicite" qu'elle fait être en étant, et qui, en étant, la fait être " (Castoriadis 1975 : 307). La caractérisation du rapport de «l'idéologie russe » à l'espace et au temps comme catégories spécifiques d'une hiérarchie de valeurs et d'un mode d'historicité particuliers peut servir de fondement au projet comparatif, et ce grâce à la reprise critique de nombreuses études historiques, 
anthropologiques et linguistiques. Le détour par une clé heuristique particulière, la notion de "souveraineté", née dans la matrice théologicopolitique propre à l'Occident (Beaud 1996), met en question le passage progressif, toujours localisé dans les « touts partiels » que sont les cultures, d'un mode de souveraineté "universelle" à un mode de souveraineté "territoriale», ce qui dans le cas de la Russie reste une question essentiellement problématique et irrésolue, décelable dans l'ambiguïté d'une contruction historique "en empire et non en État-nation" (Mendras $1997: 85)$.

Le rapport socialement institué au temps dans la Russie tsariste s'exprime comme un processus d'intrication permanente avec l'invisible légitimant, à travers une opposition hiérarchique entre "l'ici-bas » terrestre et "l'au-delà " éternel (I), qui ouvre sur une vocation «messianique » explicite par l'intermédiaire d'une historicisation de la Vérité divine. Cette temporalité particulière se réfléchit dans un rapport complémentaire à l'espace, qui intègre le « dehors » comme valeur subordonnée au tout, relayant une opposition hiérarchique qui cette fois articule un «ici-même » russe et un «là-bas » définissant l'Occident, de façon plus ou moins fantaisiste (les adjectifs injurieux de "latin ", "hongrois ", "suédois ", " hérétique » en constituent les substituts quasisynonymiques) (II). S’ils peuvent être distingués à un niveau analytique, « temps » et « espace " n'en restent pas moins conjoints au niveau des représentations sociales, caractérisant de façon spécifique un mode de «souveraineté universelle » russe, de type "socio-cosmique " (III), dont il reste à déterminer l'influence sous-jacente dans les multiples expressions "identitaires " contemporaines, foisonnante efflorescence qui fait signe vers la quête d'une auto-définition problématique, par «fin d'un empire, naissance d'une nation » interposées (Strada 1992).

\section{La naissance d'une tension fondatrice}

Vérité éternelle par le peuple, salut historique par le pouvoir

À partir de la classification anthropologique classique des différents types de souveraineté (tribale, universelle, territoriale) (Maine 1861), il est essentiel de préciser la position particulière de la Russie par comparaison avec l'évolution occidentale. L'enracinement du messianisme dans la conscience historique russe, perceptible dans la notion de «Moscou Troisième Rome» (Poliakov 1989), peut être considéré dans une perspective de substitution à la mission impériale byzantine dépositaire de la souveraineté universelle. Moscou a suppléé Constantinople à la tête du monde orthodoxe, recevant l'héritage de celle qui était à la fois «deuxième Rome » et « deuxième Jérusalem ». 
Ainsi se trouve posée dès le $\mathrm{XV}^{\mathrm{e}}$ siècle l'alliance du principe spirituel (Jérusalem) et du pouvoir séculier (Rome), le tout dans un contexte eschatologique - la fin du monde étant attendue pour 14921 . L'héritage de Byzance comme entité théocratique, "royaume de Dieu sur terre», dont la légitimité est fondée sur la préservation de la Vérité orthodoxe, tend à isoler la Russie des autres royaumes "impurs" car hérétiques. Le Tsar se nourrit d'une double légitimité, autorité sainte et pouvoir royal : il s'unit à son peuple sous le vocable intégrateur de "terre russe", il l'incarne comme maitre de l'univers entier, confessant la foi véritable qui seule donne droit à une existence authentique. L'héritage byzantin nourrit la perception chrétienne médiévale de la manifestation de la Providence divine dans l'ici-bas, justifiant par là-même la place de la Russie dans l'histoire du monde. Le Tsar est «Dieu terrestre» (Zemnoi Bog) (Schaub 1992), tout comme Dieu est "Tsar divin » : la séparation des principes spirituel et temporel qui eut lieu en Occident, et qui d'une certaine manière favorisa le passage d'une souveraineté universelle à une multitude de souverainetés territoriales, ne se réalisa pas en Russie, ou, tout du moins, d'une manière bien différente.

\section{Disjonction du Tsar-punisseur et de la Sainte Russie}

Il est en effet possible de noter dès le règne d'Ivan IV le Terrible au XVI ${ }^{\mathrm{e}}$ siècle une évolution de la place du Tsar (Goldfrank 1984 : 592)2 , occupant désormais une extranéité surplombante qui le destitue progressivement de son rôle de médiation sacrale, tout en conservant le vocabulaire traditionnel d'imbrication ordonnée dans la hiérarchie de l'Être. Alors que dans un premier temps, le Tsar et son peuple étaient Un, avec possibilité de séparation à un niveau subordonné de la hiérarchie de valeurs, dans un deuxième temps, le Tsar se retrouve du côté de Dieu, par analogie de position, face au

1. Lotman \& Ouspenki 1990 : 310. La conception de "Moscou Troisième Rome" apparaît dans un contexte eschatologique particulier à la fin $X^{e}$ siècle, puisque l'on approche des 7000 ans depuis la création du monde. Or « le septième millénaire, c'était le septième jour cosmique, le sabbat du Seigneur sur lequel l'histoire se termine, le temps où, selon la prophétie, on attend "des cieux nouveaux et une terre nouvelle" (Isaïe) ». La création du monde étant située en 5508 avant la Nativité du Christ, 5508+1492 = 7000, Ouspenski effectue un rapprochement intéressant avec l'arrivée de Christophe Colomb au "Nouveau Monde", "découverte" qui prend une signification apocalyptique d'accomplissement des prophéties d'Isaïe et de saint Jean.

2. La théorie sociale et politique de Joseph de Volokolamsk accepte les structures en place comme une donnée "naturelle» et confere au Tsar, en possession de qualités divines autant qu'humaines, un rôle « répressif » semblable à celui du père abbé au sein du monastère : "Le désordre et l'impiété peuvent ruiner le monastère comme le royaume, aussi la discipline monastique et l'inquisition généralisée sont-elles nécessaires. En outre, la prospérité sur terre découle de l'observance de la loi divine, qui procure ainsi le meilleur des deux mondes. » 
peuple dont il convient d'assurer le salut, s'il le faut en le punissant ${ }^{3}$. Le Tsarpunisseur, selon une " absorption de l'humain par le divin " dans la personne même du Christ, s'oppose désormais à la "Sainte Russie » (Besançon 1967) souffrante et pécheresse. Nous avons ici un début de distinction entre le pouvoir désormais explicitement "transcendant", d'une part, et, d'autre part, un principe disjoint, source de légitimité : le "pays ", "la sainte terre russe » et ses habitants. À terme, après un long processus non pas de "sécularisation" (puisque l'on ne quitte pas l'univers de l'Un ontologique) mais de basculement interne, s'élabore une tension entre un État à souveraineté "territoriale» (de clôture nationale), "occidentalisé", «rationnel », " éclairé», "administratif» voire "policé» (Raeff 1982) et le détenteur "sacré " de la vocation universelle, c'est-à-dire le Peuple dépositaire de la Vérité éternelle et donc identifié à l'Église.

Toutefois, si l'ancienne position sacrale « rédemptrice » est abandonnée, la relation entre le Tsar et son peuple reste au niveau d'un rapport de médiation en vue du "salut ", relation qui interdit toute fermeture de la sphère terrestre par adéquation du corps politique à lui-même comme en Occident. La hiérarchie de valeurs russe conserve, en situation prééminente, une ouverture ultime sur l'au-delà qui la fonde : l'espace social reste donc essentiellement structuré selon un principe de relations et de dépendance sociocosmique. Le monde n'a pas à être investi pour être transformé en vue du salut, tel qu'une partie du christianisme occidental le fera comprendre, mais vécu sur un mode d'expérimentation de l'humaine condition pécheresse, mode qui prohibe toute réhabilitation de la nature de l'homme, pensable quand sa raison se perçoit comme pont avec la Raison divine.

\section{Deux principes “divins" porteurs de temporalités contradictoires}

La disjonction des deux sphères, «spirituelle » et «temporelle », offre en Occident un espace de légitimité autonome au pouvoir politique, appelé à prendre en charge la sacralité collective de l'accomplissement terrestre face à une Église vouée à la sacralité des "fins dernières». Le retrait de Dieu dans une transcendance ultime - il devient l'Autre de ce monde et ne se communique plus aux hommes en tant que Destin collectif consacre l'autonomie d'une sphère terrestre désormais pourvue d'une suffisance ontologique propre. A contrario, l'évolution historique de la Russie

3. Voir l'échange épistolaire entre Ivan le Terrible et le prince André Kourbski en exil : tirant conséquence de l'illégitimité qui frappe le pouvoir royal, qui « néglige les traditions de la Russie, gouverne de façon cruelle et arbitraire contre l'avis de la noblesse et réduit son peuple en servitude», le prince Kourbski va attribuer la part divine qui traditionnellement résidait dans le Tsar à une nouvelle réalité sacrée : la "Sainte Russie ", trahie par l'Antéchrist qui tâche du sang des innocents le pays et l'orthodoxie (Szamuely $1976:$ 45). 
nous montre certes une transformation du lien religieux, mais celui-ci maintient le principe ultime de correspondance avec la présence divine. Par les actions du Tsar, véritable dépositaire de la légitimité sacrale héritée du basileus byzantin, Dieu continue à se communiquer aux hommes, non pas bien sûr en essence, mais par ses énergies déifiantes, selon la spiritualité orthodoxe théorisée par la synthèse palamite ${ }^{4}$ (voir Meyendorff 1959 et Ouspensky 1982). Dieu ne se trouve pas radicalement transcendant, mais encore visible dans le monde selon des modes de relation fluctuants et parfois «mystiques", comme les fols-en-Christ (iourodivie) qui récusent les conventions sociales (Siniavski 1990) et énoncent des vérités "divines » sous l'aspect de non-sens et de déraison ${ }^{5}$, ou les startsy (" anciens"), "pères spirituels » charismatiques dotés des dons de prophétie et entretenant des relations personnalisées avec leurs différents "enfants" (Dostoïevski, Gogol, Soloviev ou Tolstoï auront tous à un moment ou à un autre des rapports privilégiés réguliers avec un starets). Vont se généraliser, notamment par le phénomène de l'imposture (Ingerflom 1996), les rappels à l'ordre de la part d'une légitimité " populaire ", détentrice d'un principe de vérité éternelle, accordé par le passé "une fois pour toutes", envers un pouvoir politique se définissant de plus en plus dans la perspective « futuriste » d'un destin historique à assumer.

Cependant, la nouvelle économie du lien sacral ainsi présentée dans un éloignement de la figure du Tsar punisseur n'est pas sans conséquence au niveau de la perception socio-communautaire : le maintien de la médiation, devenue considérablement problématique depuis l'Incarnation du Christ (Dagron 1996), passe par une redéfinition du lieu de l'autorité. La disjonction, certes relative au sein d'une totalité socio-communautaire encore sous le signe de l'Un ontologique, entre le Tsar et la Sainte Russie, c'est-à-dire entre un principe «théologico-politique » de salut (pravda-justice, tourné vers un avenir à orientation eschatologique) et un principe de

4. Saint Grégoire Palamas (1296-1359) est considéré comme le "théologien de l’hésychasme", spiritualité de la "quiétude» (hesuchia en grec) née dans les milieux monastiques d'Égypte et de Syrie vers le IV siècle, qui privilégie «l'oubli de soi " qui conduit à l'humilité sereine et permet l'attente confiante de l'Esprit Saint. La contemplation divine se trouve favorisée par l'invocation du Nom de Jésus, liée à des techniques respiratoires. En défendant ces méthodes d'oraison psychosomatique contre certains théologiens "nominalistes" qui niaient l'expérience mystique de participation anticipée à la vie divine (le moine Barlaam, notamment), Grégoire Palamas va rédiger les Triades pour la défense des saints hésychastes, première synthèse théologique de la spiritualité des moines orientaux, qui va devenir à partir de 1351 « doctrine officielle» de l'Église orthodoxe. La distinction palamite entre essence et énergie divines permet un dépassement de la " théologie apophatique » en déclarant que Dieu, transcendant sa propre transcendance, se rend participable tout en restant inconnaissable. L' « union avec Dieu » reste donc possible, une fois l'homme par la prière revenu à sa nature déifiée par la grâce originelle d'avant la Chute: la "transfiguration » du corps et de la raison par l'Esprit permet à Dieu de se rendre réellement visible.

5. Selon M. Evdokimov (1987: 46 sq.), les «fols-en-Christ» constituent la réponse du peuple russe au pouvoir du Tsar se «sacrifiant " pour assumer la responsabilité et la charge morale du pays, car ils persistent à lui rappeler les limites terrestres et la vanité d'une telle mission. 
sens "donné» et éternel (pravda-vérité, acquise dans le passé, par la conversion de la Russie) provoque une tension extrême, dont la volonté slavophile de restauration de l'intégrité (tselnost') au sein d'une culture pleinement orthodoxe ne sera qu'un lointain avatar, une tentative de réponse définitive, certes connotée par l'influence du romantisme allemand (Christoff 1961, 1972; Gratieux 1939; Rouleau 1990; Vibert 1999; Walicki 1980).

\section{Le maintien dans l'Un ontologique}

Dans l'histoire russe $\mathrm{du} \mathrm{XV}^{\mathrm{e}}$ au XVIII ${ }^{\mathrm{e}}$ siècle, la position de médiation sacrale est occupée par la figure du Tsar, héritant du «legs byzantin » pour asseoir sa légitimité d'icône divine. Mais, progressivement, le monarque russe ne récapitule plus le message chrétien en "vivant comme le Christ » (se signalant donc par sa piété et sa participation à l'humaine condition pécheresse) mais en "agissant comme Dieu ", punissant ses sujets afin de les conduire vers le salut. Cette dimension "activiste", tout en préservant le principe de médiation, en déplace le sens : le tsar reste sans doute corps rédempteur unissant les croyants, matérialisant l'intégrale et définitive conformité de l'ordre humain à l'ordre du monde, mais il se double d'une légitimité d'intervention dans l'énonciation de la Loi, d'expression de la Présence divine. Or, en Occident, c'est la reconnaissance de la grandeur de Dieu, et le raffinement dans l'image de sa toute-puissance qui ont été les plus sûrs instruments du recouvrement par la société humaine de sa disposition d'elle-même : la distance de Dieu a permis une autonomisation croissante de la sphère terrestre, pourvue d'une consistance propre (Gauchet 1985). A contrario, la présence constante de Dieu dans les affaires des hommes au sein du monde orthodoxe, par l'intermédiaire même de ses énergies qui sanctifient les actions temporelles, maintient l'Un ontologique, l'inclusion de l'ordre visible dans un principe d'englobement supérieur. D'où ce dédoublement à partir d'Ivan le Terrible entre la figure du tsar et l'image de la Sainte Russie: assimilées sous le règne d'un tsar pieux et juste, ces deux valeurs se dissocient et s'opposent radicalement durant l'exercice du pouvoir d'un "Antéchrist». Une dichotomie entre, d'une part, un pouvoir qui est et fait être la société à certains égards, qui la contient et la résume, en un mot qui l'incarne (pouvoir qui reste le monopole du Tsar) et, d'autre part, une autorité qui se doit d'être à quelque titre l'incarnation des raisons dernières et de la loi divine, et qui se réfugie dans la notion de "Sainte Russie» (Laran \& Saussay 1975).

La tension entre les deux termes restera prégnante tout au long de l'histoire russe, sa conséquence essentielle étant l'impossible instauration d'un 
«espace neutre», d'une «zone objective» (État comme bien public, Loi comme abstraction rationnelle), appuyée sur une séparation des faits et des valeurs (Lotman \& Ouspenski 1990 : 22-23). La rupture de l'unité donnée comme éternelle (Tsar-peuple), déchirement visible à travers le phénomène de l'imposture (Ingerflom 1992), conduit à laisser le modèle artificialiste du pouvoir après Pierre le Grand ("remodeler » le peuple, l'amener à la «civilisation ») dans la dépendance d'une hiérarchie de valeurs intégratrice et dualiste, formée à partir des catégories antinomiques pur/impur, ancien/nouveau, Orient/Occident, etc. L'« occidentalisation» du régime politique se trouve réinterprétée dans un cadre religieux global, intériorisée dans une stricte opposition bien/mal qui structure la société et sa perception du monde. Alors que le principe de « l'Autre » était renvoyé à l'extérieur du social, dans une conception plus "traditionnelle", sous forme d'hérétique, de païen, pour former un élément d'opposition subordonné au tout des relations socio-cosmiques, il va être de plus en plus intégré comme élément interne de la totalité sociale, "conscientisé " par le peuple sous l'effet de l'action volontariste de Pierre le Grand. L'«Europe» ainsi reconstruite devient une part indissoluble de la culture russe, soit comme modèle, soit comme repoussoir, mais toujours sous forme absolutisée et essentialisée.

\section{Le rapport à "l'espace”}

La Russie dans le miroir de l'Occident après Pierre le Grand

"Tout le dispositif de la cour - les institutions fondées par Pierre, les réformes accomplies y compris le changement de titre (il est le premier empereur russe), la renaissance de la conception sur la Troisième Rome, le parallèle avec Constantin élaboré à son propos comme d'autres comportements du pouvoir - tend à faire apparaître Pierre comme un démiurge, et non plus comme le garant de l'harmonie sociale ou du bien public. [...] Autrement dit, les idées occidentales introduites par le pouvoir et par des méthodes anciennes se retrouvent, captées par l'archaïsme, au service du messianisme traditionnel du monarque russe» (Ingerflom 1993 : 129-130).

Les modifications engendrées par l'action artificialiste du pouvoir sur la société, si elles sont considérées de prime abord comme " externes", vont être "absorbées » par la culture d'accueil en fonction de son organisation particulière. Cette acculturation représente un "phénomène social total ", touchant tous les niveaux de la réalité sociale et culturelle. Elle peut expliquer les raisons du mouvement historique de la culture: traditionnellement inscrit dans un registre «inversé », d'hérésie, de mal, de péché et de corruption, l'Occident, qu'est censé représenter le pouvoir impérial de Pierre avec ses réformes autoritaires, va déteindre sur l'Empereur et sa perception dans le peuple. 


\section{Un Empereur à la fois "occidental" et Antéchrist}

152 Pour une partie de son peuple, Pierre I $^{\text {er }}$ va incarner non pas le Tsar sauveur, pieux et élu de Dieu, mais bien plutôt l'Antéchrist, le plus souvent imposteur, sans droits légitimes au trône impérial, s'étant substitué au vrai Tsar lors de son voyage en Europe. Boris Ouspenski nous rappelle ce témoignage "populaire » à propos de Pierre :

"C'est un Suédois qu'on nous donne pour Russe, c'est pour cela qu'on baptise et qu'on marie à l'envers et qu'on peint des icônes dont les personnages ont des têtes de Suédois, qu'il ne peut jeûner ni faire maigre en carême, qu'il aime le vêtement suédois, qu'il mange et boit avec les Suédois et ne quitte pas leur royaume [...] que le grand duc Pierre Alexeevitch est né d'une Suédoise et qu'il avait déjà des dents en naissant, c'est donc l'Antéchrist» (Platonida, femme âgée réputée pour sa sagesse, citée in Lotman \& Ouspenski 1990 : 361-362).

Antéchrist, Pierre est donc initialement un imposteur: des rumeurs de substitution, à l'étranger ou durant sa prime enfance, s'étaient répandues avec insistance. D'où une série d' "authentiques et véritables Pierre" mais également de personnages incarnant le tsarevitch assassiné, Alexis, émergeant du peuple pour répondre à la légende selon laquelle le vrai Pierre avait été tué lors de son enlèvement en Europe pour être remplacé par l'Antéchrist sur le trône. Ce phénomène de "l'imposture » marque durablement les représentations et modes d'action populaires à partir de Pierre

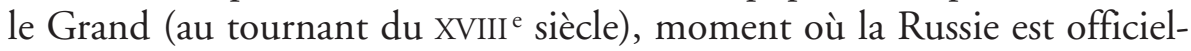
lement en voie "d'occidentalisation» et de «civilisation». Ce n'est bien évidemment pas une coïncidence et cela souligne que l'évolution effective du régime tsariste vers un mode de domination en terme de "pouvoir» (légitimé par le "Bien public») se conjugue historiquement avec la persistance d'une compréhension "traditionnelle » de l'autorité dans la hiérarchie de valeurs populaires, qui a pour effet non seulement de récuser la légitimité des autorités en place, mais également de favoriser l'apparition de "pratiques restauratrices", visant à nier un dualisme vécu comme pathogène (entre Bien et Mal, Russie et Occident, "vraie foi » orthodoxe et hérésie, Tsar sacré et Antéchrist).

\section{Le rapport au Tsar comme pouvoir de la société sur elle-même}

Dans son étude sur les mouvements utopiques à caractère social apparus en milieu paysan, Kirill Tchistov (1996) a relevé trois types essentiels : le "siècle d'or", où les idéaux utopiques sont projetés dans un passé mythique, les "terres lointaines" qui permettent de les situer dans un espace " autre", et enfin les légendes sur les "sauveurs", notamment des 
tsars, auxquelles Tchistov (ibid.: 630) rattache le phénomène historique de l'imposture. Selon lui,

«... la longue existence du servage, la dépendance étroite à l'égard des propriétaires

terriens, liée à la centralisation excessive de l'appareil d'État (autocratie) ont fait naître la croyance en la possible intercession du tsar, lequel se retrouvait placé au-dessus de la pyramide féodale et semblait en être indépendant ».

Les légendes sont généralement liées à un tsar ou tsarévitch (dauphin), écartés du trône de façon jugée illégitime, suivant le schéma défini par Tchistov: «le tsar assassiné (ou le tsarévitch en exil) n’avait en réalité pas péri, il avait pu échapper à ses assassins, il était resté vivant, s'était caché et, au moment voulu, il viendrait car il devait libérer le peuple (c'est pour cela qu'on avait voulu le tuer). Ceux qui prendraient son parti seraient les premiers à recouvrer la liberté et seraient distingués par lui » (ibid.).

Au cœur de ce dispositif de "l'imposture» se trouve une réflexion du tout social sur lui-même et sa représentation : jamais le peuple ne se pense ici comme étant à l'origine du pouvoir, par un quelconque mode de désignation ou de "représentation" (au sens moderne). Pourtant, le tsar pensé comme légitime ne peut "que" rejoindre les aspirations paysannes, et pour ainsi dire se confondre avec elles. Au sommet de la hiérarchie de valeurs, le tsar "juste " et la société ne font qu'un. Le tout bien sûr en relation avec la volonté divine qui sous-tend cette «incarnation» terrestre. Nous avons ici ce que Marcel Gauchet (1981 : 149) nomme «la dette religieuse du sens » qui détermine " une double conjonction : de l'un individuel du pouvoir avec la multiplicité collective, et, en la personne du pouvoir, de l'ici-bas et de l'au-delà ». Il faut arriver à penser que, "en privant un monarque de sa légitimité, le social s'autorise la révolte et autorise l'un des siens à s'auto-nommer" (Ingerflom 1992: 163) et, en même temps, que «le lieu depuis lequel la société est capable de se penser, de se pourvoir de sens et d'agir sur elle-même est hors de la société " (Gauchet 1977 : 21). Il s'agit d'une "hiérarchie de valeurs", «à la fois intérieure et extérieure au tout social que constitue chaque société » (De Coppet 1998 : 161). La noblesse est récusée par la paysannerie en tant que pouvoir intermédiaire falsifiant et déformant les pensées du tsar et ses volontés de libération du peuple. Le monarque juste, «élu de Dieu », se confond avec son peuple porteur de la vérité divine, laissant entendre que le tsar injuste aura été nommé par le Diable. La disjonction entre l'imposteur et le pays, la "Sainte Russie", est alors patente: "Tous de leur propre volonté se sont soumis à lui qui est en dehors de la terre russe, et lui qui est une idole, on s'incline devant lui comme devant un tsar", dit le chroniqueur (Ivan Timofeev dans son Vremmenik, à propos d'un "faux-Dimitri», cité in Lotman \& Ouspenski 1990 : 337). Le peuple ne se perçoit que sous la 
figure d'un Tsar juste, éternellement fidèle aux préceptes divins, et ceci

quels que soient les «tsars", porteurs contingents du pouvoir mais dont la légitimité est suspendue à la volonté divine - celle-ci étant décelable en partie dans leurs actions terrestres.

"L'occidentalisation », la rationalisation du pouvoir étatique, loin d'aboutir à la constitution d'une sphère "légale» dans les perceptions populaires, d'une séparation entre la figure du monarque, naturelle, et la position du pouvoir, sacralisée, les laisse dans l'indifférenciation: un tsar légitime et injuste est inconcevable. L'État bureaucratique, "moderne", avec son administration et son armée, qui se construit à partir du XVIII ${ }^{\mathrm{e}} \mathrm{siècle}$, se traduit donc dans le langage populaire, notamment parce que ses représentants sont issus de la noblesse, en "royaume du Malin ", imposant une coupure entre le tsar pieux et ses sujets, entre le Tsar et le Peuple comme les deux hypostases d'une même réalité idéale, "l'Idée russe" énoncée dans la hiérarchie de valeurs de la totalité socio-communautaire. Au niveau subordonné, la "Sainte Russie ", comme terre sanctifiée par Dieu, s'oppose au tsar vivant, oscillant entre "icône divine" et "figure de l'Antéchrist ". L'ordre social n'est pas d'ici : toute révolte, révolution, tout changement radical, ne s'institue qu'à partir de son contraire, une hiérarchie de valeurs portée vers l'éternité, l'immobilité, l'universalité, l'unité, la vérité. L'aspiration à la restauration, le respect d'une tradition sacrée seuls permettent une redisposition de l'organisation collective, transformation qui reste impensée au regard de l'origine divine du pouvoir, inaccessible en principe aux agissements humains. Est-ce trop audacieux de rapprocher cette perspective de ce qui nourrira la «logocratie» marxiste-léniniste : une obéissance consciente à des "lois ", naturelles, historiques, une Providence qui légitime l'intervention humaine en la rendant cohérente avec son propre destin? Un artificialisme conçu comme mise en adéquation parfaite à une Histoire extérieure au pouvoir des hommes, la conjonction du donné et de l'advenir: ne reste qu'un ordre foncièrement immanent dans sa dénégation de tout invisible institué, et parfaitement transcendant dans son rapport au social qu'il entend réformer. "Immanence» et «transcendance» ne sont donc plus que des points de référence, les deux extrémités de l'échelle moderne qui nous sert à appréhender, bien difficilement, l'auto-compréhension d'un tout social : la hiérarchie de valeurs est un ordre "socio-cosmique", compris au sens d'idées-valeurs et d'actions, qui défait au niveau de référence ultime les contrastes binaires, platement dichotomiques et contradictoires élaborés selon notre conception moderne du tiers-exclu ${ }^{6}$.

6. Ainsi, parmi d'autres, les difficultés inhérentes aux oppositions spirituel/matériel, idée/valeur, sujet/ objet et bien d'autres, voir De Coppet 1998: 165, note 12. 


\section{Une souveraineté universelle au fondement "socio-cosmique"}

\section{Souveraineté universelle, souveraineté territoriale}

Nous posons ici l'hypothèse que toute idée particulière de "souveraineté " s'instaure à partir de la mise en forme "politique" des relations interdépendantes au sein d'une totalité sociale entre conceptions de l'espace (territoire, localité) et du temps (temporalité traduite en termes sociaux). En cela, l'idée de souveraineté territoriale d'origine moderne, fondée sur l'étendue et la clôture d'une collectivité pourvue de volonté et de conscience immanente, s'oppose à la notion de souveraineté universelle $e^{7}$ dont le principe est historiquement déposé dans un passé mythique à reconduire, voire à réaliser.

Si l'on veut définir deux idéaux-types, nous avons d'un côté la perception "holiste", hantée par un récit ontologiquement originel permettant de lire de manière essentiellement cyclique et "socio-cosmique" la suite des événements, et pour qui la dimension « locale " particulière n'apparaît que secondaire, subordonnée à une intégration hiérarchique dans la chaîne de l'Être. De l'autre côté, la conception « moderne » s'élabore à partir d'une "conscience historique " linéaire et causale, orientée vers un Progrès: l'historicité évacue la distinction traditionnelle ordre/chaos, hommes/non-hommes pour délimiter un espace social en pleine possession de son principe (généralement contractuel) par auto-réflexivité. L'innovation chrétienne permet le passage d'une extériorité temporelle (l'altérité radicale du fondement exigeait une permanence intangible de l'ordre socio-cosmique, à reconduire par les rituels) à une extériorité spatiale (l'homme a désormais accès au fondement en déchiffrant le Sens de la Création : Dieu devient "transcendant ", c'est-à-dire éloigné dans l'espace mais présent à tout moment dans le cœur du croyant). Dans les deux cas certes, le particulier (local) fait signe vers l'Universel, mais d'un côté, celui-ci est donné selon une hiérarchie de l'Être qui préserve et intègre une diversité ordonnée (seuls les membres du social sont véritablement " humains ", mais " être homme» constitue seulement un niveau relatif au sein de la totalité socio-cosmique globale), alors que de l'autre, il doit proprement advenir, c'est-à-dire résorber progressivement les «imperfections » particularisantes par l'avènement de l'Un parfait. La souveraineté «territoriale» ne sera possible qu'une fois cet Universel compris comme enraciné dans une collectivité particulière, incarnant «la» Civilisation

7. «... la notion moderne de souveraineté exprime une idée de suprématie, tirée d'une laïcisation du pouvoir divin, et qui témoigne donc d'une vision superlative du pouvoir. [...] l'État moderne saisit indifféremment les individus dès lors qu'il se trouve sur son territoire. À l'hétérogénéité de l'espace politique romain s'oppose l'homogénéité de l'espace politique étatique» (Beaud 1996: 627). 
comme processus de l'humanité en marche vers la réalisation effective de soi. Autrement dit, il a fallu que l'Universel subisse potentiellement une extension illimitée, intégrant l'ensemble du genre humain sous la forme d' "individus ", par-delà toute appartenance communautaire, pour que cet héritage de "l'individu-en-relation-directe-avec-Dieu» recompose le vivre-ensemble sous la forme d'individus collectifs homogénéisés (en l'occurrence les nations modernes), après destruction de la pluralité des statuts sociaux hiérarchisant les totalités holistes.

\section{L'institution "socio-cosmique" du mir}

Cette distinction une fois posée, et pour revenir sur le cas russe, il faut insister sur la prégnance historique d'un socio-cosmisme à orientation "universelle», particulièrement appréhendable dans l'institution de la commune paysanne, le mir. Fondé sur la propriété collective du sol, opérant par redistribution périodique des terres sur décision d'un conseil réunissant les pères de famille, le mir fut véritablement le "monde " (autre sens du terme mir) du paysan russe. L'État, la commune et la famille sont souvent vus comme trois anneaux constitutifs d'une même chaîne possédant une analogie de principe. Tout comme la famille, la commune rurale se définit selon deux traits essentiels : l'autorité illimitée du père et la propriété indivise entre les enfants. La famille, abritant souvent plusieurs fils mariés et ménages collatéraux sous la direction de l'aïeul, constituait une «commune en miniature». Les femmes, du point de vue de la propriété, ne font pas partie intégrante de la maison ou de la communauté : la fille n'est que l'hôte temporaire de la maison paternelle qu'elle doit quitter lors du mariage. En revanche, il est permis aux jeunes femmes d'accumuler un petit pécule personnel (par de menus travaux ou des économies : la "corbeille») qui lui servira de dot au moment du mariage. En échange, le fiancé s'acquittera des frais de noces, souvent considérables. Si un fils sort de la maison commune de son propre chef, le père est libre de ne rien lui donner, le laissant partir « avec la croix seule " (c odnim kriestom $)^{8}$.

Le mir en tant que structure sociale peut être défini comme le lieu d'incarnation du principe de sobornost, idée-valeur véhiculant les sens de " communauté », " conciliarité », " catholicité » (au sens originel), attachée au corps mystique de l'Église. La hiérarchie de valeurs russe intègre de façon progressive une analogie entre Église (sobornost' comme communion ecclésiale), commune rurale (mir comme lieu de vie de la fraternité com-

8. On peut poser l'hypothèse que le fils partant "avec la croix seule" s'apparente pour un temps à un " errant ", c'est-à-dire un "individu-en-relation-directe-avec-Dieu " (par "la croix»), en rupture avec l'ordre social de continuité et de permanence institutionnalisé dans la communauté du mir. 
munautaire, des valeurs morales de dévouement, générosité et sacrifice) et peuple (narod). Selon la conscience russe, la Vérité chrétienne, préservée dans l'Église orthodoxe, se perpétue donc dans le mode de vie des communautés paysannes et, par extension, dans la réalité nationale elle-même : ici se joue sans conteste un mode de relation étroit avec ce que nous avons défini comme "souveraineté universelle». C'est dans ce sens que le mir et la Russie sont compris dans un ensemble de relations socio-cosmiques qui les relie à la terre, selon une perception particulière de la dimension spatiale, étrangère aux caractéristiques de la "souveraineté territoriale».

\section{Terre-Mère et Sainte Russie}

L'homme n'est pas perçu comme pouvant dominer la terre, mais comme son fils : il nait de celle-ci et finit par y retourner. La terre est ainsi une mère charitable, pleine de compassion pour les pécheurs et souffrant avec les déshérités, mais dans le même temps, elle reste "une source inépuisable de force et de santé : elle donne des plantes qui guérissent les maladies humaines et elle-même guérit, avec le temps, tous les malheurs " (Spidlik 1994: 199). Blessée par le mal répandu par les hommes, elle cherche à purifier les pécheurs et prie pour eux, d'où la pratique de la confession à la terre de ses propres péchés. Nombre d'auteurs ont souligné le lien évident avec les rites préchrétiens de la Terre-Mère nourricière (Mat'Syra Zemlia), fécondée par la pluie pour devenir mère9 ${ }^{9}$ La mort est donc vue comme un simple retour au sein maternel qui a enfanté l'homme. Symbole de pureté, la terre est censée rejeter les cadavres des criminels et des sorciers après leur sépulture ${ }^{10}$. S'agenouiller afin d'embrasser la terre constitue un geste répandu parmi les chrétiens russes, qu'il s'agisse de se défendre d'une accusation calomnieuse ou bien de renforcer un serment (dans certains cas gravissimes, elle est même mangée) : Dostoïevski décrit Aliocha Karamazov baisant la terre dans un moment d'extase et ressentant l'amour de Dieu pour l'ensemble du règne vivant.

La Terre-Mère humide est souvent apparentée à la Mère de Dieu, en tant que protectrice et consolatrice du genre humain. La Mère de Dieu se trouve

9. Iouri Lotman et Boris Ouspenski ont étudié le rapport entre les mots obscènes ou jurons d'une part, et les offenses faites à la Terre d'autre part. Ainsi, le mandement contre ceux qui jurent dans la Russie traditionnelle, parfois attribué à Jean Chrysostome, est particulièrement explicite dans le lien qui est fait entre les trois "mères" de chaque homme : "Il est inconvenant pour un chrétien orthodoxe de jurer. À cause de la Très Pure Vierge Mère de Dieu [...] car par elle nous avons connu le Fils de Dieu [...] deuxièmement, de la deuxième mère, chère à tout homme, par laquelle nous sommes venus au monde. Et la troisième mère, la terre qui nous fournit la nourriture et abondance de biens et à laquelle, sur l'ordre de Dieu, nous retournerons pour y recevoir la sépulture " (Lotman \& Ouspensky 1990 : 400-424).

10. Zelenin 1994. Ses travaux (réalisés entre 1910 et 1940 environ) sur les morts "impurs " (zalojnyie) comme les suicidés, les sorciers, les pécheurs, les non-orthodoxes (en fait, tous ceux que l'on ne peut inhumer parce qu'ils souillent la terre) constituent encore aujourd'hui une source d'une richesse inépuisable pour les recherches ethnographiques et viennent d'être réédités en Russie. 
être non seulement la mère du Christ mais également celle de tous et de tout : sa maternité s'étend à l'ensemble de la nature et son image s'interpénètre ainsi avec la Terre-Mère humide. Tout en étant formellement distinguées, les trois mères que sont la Mère de Dieu, la Terre-Mère humide et la mère de chaque homme s'avèrent structurellement assimilées dans leurs fonctions de maternité et protection. Au cours de l'histoire russe, le concept mystique de "Sainte Russie " va progressivement être associé à ce binôme Mère de Dieu/Terre-Mère : "La Russie est la Terre dont dépend le salut de l'humanité, de même qu'au moment de l'Annonciation, tout a dépendu de l'acceptation de Marie» (Besançon 1967 : 130). Le terme même de "Sainte Russie» (Sviatorousskaia zemlia, littéralement " terre sainte-russe»), s'il est d'utilisation rhétorique explicite récente (fin XVI siècle sous le règne d'IvanIV le Terrible, dans les lettres au Tsar de l'opposant au régime exilé en Pologne, le prince André Kourbskii, afin de parler du pays qui souffre le martyre sous la tyrannie sanglante d'Ivan), semble provenir des profondeurs des traditions populaires : la Sainte Russie existe quelque part au fin fond de la Russie réelle, dans un espace ou un temps autre. Dès l'origine, le trait dominant du christianisme russe a été l'accent mis non pas sur l'appel du Christ à chaque individu mais sur la conversion du pays tout entier, qui représente une véritable occasion de prise de conscience collective (Dennes 1987 et Vodoff 1988). L'héritage byzantin donne d'ailleurs un sens à l'histoire de la Russie, dont la christianisation est justifiée par l'imposition d'un décret providentiel divin. Le concept de «Sainte Russie» dans le peuple a très tôt été confondu avec la Palestine, comme Terre Sainte, ainsi que l'attestent les récits contant l'histoire de saint Georges (Gueorguy, Youri) qui, dans les hymnes russes, se trouve être le fils de sainte Sophie (la Sophia incarnant la sagesse de Dieu), "né à Jérusalem en terre russe " ${ }^{11}$. La foi chrétienne engage non seulement les humains, mais également la terre russe et la nature, baptisées du même coup. Pendant la Semaine Sainte, le Christ parcourt la Terre russe, soit sous une forme invisible, soit sous le déguisement d'un pauvre mendiant, qui permet au peuple de s'apparenter au Christ ( La confrérie des indigents incarne en quelque sorte la figure du Christ russe », Siniavski 1990 : 208). Lidéal de "Sainte Russie » a implicitement dominé la première partie du règne d'Ivan IV le Terrible, entre 1547 et 1560, légitimant un immense travail d'unification de la foi, des mœurs, de l'histoire,

11. La légende de saint Georges est relatée par Siniavski (1990 : 241-242). La suite est tout aussi intéressante puisque, enlevé par des infidèles selon la légende, le saint s'évade et rétablit partout en Russie la foi chrétienne, non seulement dans le monde humain mais au sein même de l'univers naturel : il transforme véritablement le chaos en cosmos et institue l'ordre divin sur terre, commandant aux montagnes, forêts, rivières et animaux. Ce motif cosmogonique est à rapprocher de la restauration de la tselnost' de l'homme, qui dépasse sa propre personne puisque son statut d'imago Dei lui permet par là-même de transfigurer le cosmos selon la grâce divine. 
afin de "fixer le vieux temps" (starina) exemplaire menacé par l'influence étrangère (Heller \& Niqueux 1995), canonisation de saints locaux, concile des cent chapitres (Stoglav en 1551), composition du livre des degrés (liste des monarques russes depuis... l'empereur Auguste de Rome!) et du Domostroï (le "Ménagier» moraliste du moine Sylvestre, modèle d'économie domestique pour une société patriarcale pieuse et ritualiste).

\section{Errants et vagabonds mystiques : la quête de la "patrie céleste"}

L'antinomie entre les deux attitudes devant le monde qui se perpétuent à travers l'histoire chrétienne - aimer la nature comme création de Dieu ou la rejeter comme indélébilement marquée par le sceau du péché originel dans l'attente de la parousie - trouve sa résolution dans la doctrine de sanctification de la nature visible élevée vers le niveau supérieur d'une vie transfigurée, "théophanique» (N. Lossky), ainsi que l'affirme cette formule de Paul Evdokimov: "Consacrer le monde, c'est le forcer à passer d'un état démoniaque à l'état de créature consciente de Dieu " (Spidlik 1994 : 210). L'une des caractéristiques essentielles de la spiritualité russe qui traduit cette volonté de divinisation du tout, rétablissement de l'intégrité perdue de la nature, est le strannitchestvo, mode de vie du pèlerin, expiant les péchés du monde par son existence exemplaire. On connaît les fameux "Récits d'un pèlerin russe »: le "vagabond mystique " fut l'une des figures archétypales les plus constamment reprises de la littérature russe. Le nombre massif de kaliki (infirmes) et perexojie (ambulants) circulant sur la terre russe atteint un tel niveau que parfois le pouvoir dût prendre des mesures limitatives. Par ses déplacements, le peuple russe cherche sans doute "une image idéalisée de la Sainte Russie ", inspirée de très loin par un goût immodéré des pèlerinages. Souvent, hommes et femmes appartenant à toutes les couches de la société russe rompaient un temps avec leur famille pour se mettre en route. La Palestine et Constantinople constituaient évidemment les destinations privilégiées des pèlerins, mais de multiples lieux saints en terre russe étaient également très fréquentés, comme la Laure de Kiev, la Laure de Zadonsk (où se trouve la tombe de saint Tikhon) ou la ville de Sarov, qui conservait les reliques de saint Séraphim. Le pèlerinage se traduit par la quête d'un sanctuaire vénéré, d'une terre promise plus spirituelle que physique, d'un idéal de perfection, à partir de cette "nostalgie de l'espace ", souvent rattachée à la mentalité collective russe, l'immensité de la steppe rejoignant celle du ciel pour incliner l'homme à la méditation intérieure ${ }^{12}$.

12. Pour Michel Evdokimov (1987 : 9), l'homme russe tend à transformer «l'infini de l'horizon en catégorie spirituelle». 
La mythique Sainte ville de Kitèje, devenue invisible lors de l'invasion tatare et située selon la tradition aux alentours du lac Svetloïar (elle s'y reflète dans les eaux), constitue en quelque sorte l'incarnation idéale de cette Sainte Russie : des lettres envoyées de Kitèje circulent dans les villages russes pour décrire le modèle de perfection chrétienne vécu dans la Ville sacrée (Heller \& Niqueux 1995: "L'utopisme populaire»). Lors du schisme des vieuxcroyants, Kitèje représentera le symbole de l'Église invisible des raskolniki contre l'Église officielle nikonienne. Au même moment (mi-XVII siècle), il ne circulait pas moins d'une douzaine de légendes affirmant l'existence d'un millenium quelque part en Russie, élément qui constitua un facteur essentiel, bien que souvent négligé, du mouvement de colonisation russe vers l'est, la recherche de terres "libres", dites " terres blanches ».

La vocation de l'homme de foi errant serait donc de rejoindre sa véritable patrie, céleste, en parcourant sans but précis les chemins terrestres. La relation entre la "spiritualité de la route» et l'idée-valeur de tselnost' ( intégrité », liant l'homme à son fondement divin d'avant la Chute) se trouve dans l'aspect socio-cosmique de cette dernière qui dépasse le monde proprement humain pour donner en modèle un type d'individu au-delà non seulement des conventions sociales de son environnement, mais surtout des contraintes psychophysiologiques liées à sa nature temporelle. «Le point de départ de la mystique du cheminement est la découverte que la connaissance de Dieu ne se laisse pas renfermer dans les limites des notions de la raison" (Spidlik 1994: 212). L'errance apporte l'expérience de la Providence divine, redonne valeur à la contemplation de la nature et initie l'homme à la "prière cosmique", œuvre de rassemblement, de réunification des agrégats de l'âme dispersés par la fuite dans l'action et la recherche du plaisir individuel, maladie ontologique de l'être déchu. Se libérer de son inscription terrestre, territoriale ou familiale, équivaut à souligner la déficience constitutive du monde d'ici-bas et l'imperfection de l'homme en quête de Dieu. L'évolution cosmique est une incarnation progressive du divin dans l'homme et la nature créée, par laquelle Dieu achève sa création et par là-même la justifie. Le baptême de la Russie importa le christianisme byzantin «sous une forme eschatologique et maximaliste» (Heller \& Niqueux 1995: 15), comme un "appel à la perfection ", à la "sainteté ", et non comme un système de morale déterminé. Le salut de l'homme ne sera jamais vu comme individuel mais s'inscrit uniquement dans la foi en une restauration paradisiaque universelle, l'apocatastase, au titre que «Dieu est devenu homme pour que l'homme devienne Dieu ». D'où de multiples formes marginales de piété populaire qui vont redoubler l'Église officielle de plus en plus engagée dans le soutien au pouvoir autocratique du Tsar comme 
unique représentation de la Providence divine sur terre: millénarismes et messianismes, qui malgré leur surnom de sectes "rationalistes", ne vont jamais "privatiser" ou "subjectiver" la croyance, tel le mouvement de Réforme en Occident, mais bien au contraire développer des espérances "chiliastes", c'est-à-dire attentes d'un bouleversement radical et global instaurant un "Royaume de Dieu sur terre", simplement parce que la sobornost' universelle, la Sainte Russie, ne se trouvait plus incarnée par le détenteur du trône sacré, devenu lieu d'imposture, le plus souvent légitimation du règne de l'Antéchrist.

\section{La Sainte Russie, médiation entre mir et cosmos}

Dans la Russie traditionnelle, le mir identifie donc la communauté locale au monde, et ces deux différents aspects de la même réalité à la Russie, par l'intermédiaire de l'orthodoxie comme Vérité universelle et éternelle. Se perçoit une analogie structurale avec le triptyque qui relie la mère de chaque individu (le " local » comme lien d'inscription individuelle dans la continuité des générations) et la Terre-Mère (le "monde " non seulement terrestre mais "socio-cosmique ") par le lien à la fois unificateur et déterminant (car hiérarchiquement prévalant) de la Mère de Dieu, matrice du processus de sanctification de l'ici-bas (hommes, terre et cosmos) dans l'acceptation de l'essence divine qui est sa nature réelle. Si l'on rapproche ces deux ensembles de relations trinaires, la séparation local/ universel ( $\mathrm{mir} /$ monde et mère individuelle/Terre-Mère) se trouve surmontée au niveau ultime par son intégration dans un « registre " divin de transfiguration théurgique, à la fois «temps» et «espace»: la Russie "messianique» comme inscription spatiale et principe temporel d'une Histoire qui s'avère théophanie.

\section{Une matrice théologico-politique originale}

Par le terme de "relations socio-cosmiques", il est donc plus aisé de décrire la compréhension du temps et de l'espace au sein d'une hiérarchie de valeurs qui, à un certain niveau prééminent, ne sépare pas ces notions, ce qui ne veut pas dire qu'elle ne les distingue pas. Toute société est en effet instauration d'une "temporalité » et d'une «spatialité » implicite: "chaque société est aussi une manière de faire le temps et de le faire être, ce qui veut dire : une manière de se faire être comme société " (Castoriadis 1975: 307). Cette constatation de Cornelius Castoriadis à propos du temps vaut aussi pour l'espace qu'elle "fait être", et donc qu'elle est. La particularité de la Russie "traditionnelle" consiste non pas en son caractère essentiellement porté à la souveraineté universelle (il est possible de dire que c'est le cas de 
l'ensemble de sociétés " non modernes ») ${ }^{13}$, mais bien en la manière originale dont elle constitue et est constituée par ce mode de relation entre local et universel. Nous avons souligné l'analogie structurale qu'il est possible de déceler dans les triades mir/monde/ «Sainte Russie », d'une part, et mère de chaque homme/Terre-Mère/Mère de Dieu, d'autre part. La tension essentielle entre les deux premiers termes, le local et l'universel, l'homme individuel et la "nature" (nous pourrions ajouter dans un vocabulaire « moderne » : le sujet et l'objet) se résout à un niveau supérieur selon le troisième terme qui englobe la contradiction précédente et la "comprend» (aux deux sens de l'expression: elle l'intègre et en «élucide» le sens global) : la "Vérité incarnée » relie et unifie les deux aspects sans pour autant les fusionner et les faire disparaître. Le troisième terme non seulement intègre les deux premières notions qui sont distinguées à un niveau subordonné mais luimême garde la trace de cette dichotomie surmontée. Tant la "Sainte Terre Russe " que la "Mère de Dieu» font signe dans la composition de leur expression (" terre ", « mère » comme partie du monde terrestre, "Sainte » et «Dieu» du monde céleste) vers les deux aspects qu'elles ont à transfigurer et à incarner : l'humain et le divin, le visible et l'invisible, l'ici-bas et l'au-delà.

La caractéristique de «l'universel russe» est de se situer constamment dans un processus eschatologique de transfiguration divine, non seulement pour le monde terrestre connu, mais surtout pour le Cosmos et l'univers dans sa totalité. Si le christianisme dans sa dynamique essentielle représente bien « une valorisation du Temps historique» (Eliade 1965: 98) au détriment du temps cosmologique, il est particulièrement frappant de remarquer que, dans le christianisme russe, l'épaisseur de la dimension eschatologique impose la prégnance de cette perception "cosmologique", où le temps "n'est jamais pensé séparé des événements qui le remplissent» (Lotman \& Ouspenski 1990 : 306). Boris Ouspenski l'a montré à propos de la conception "Moscou Troisième Rome» qui, tout en possédant également un fondement "historique " (ou "moderne », c'est-à-dire le lien, par un processus de causalité linéaire, à des événements immédiatement antérieurs dans le temps, à savoir la chute de l'Empire byzantin en 1453 et le rejet définitif de la domination tatare en Russie vers 1480 : la chrétienté triomphe de l'islam en Russie au moment même où l'inverse se produit à Byzance), se voit attribuer une valeur cosmologique, avec l'accent mis sur "l'aspect cyclique » (ibid. : 314) - avec Moscou comme «nouvelle ville de Constantin", donc

13. Eliade (1965: 66-69) remarque que dans de nombreuses sociétés traditionnelles, il y a solidarité et interdépendance entre espace ("le Monde») et temps ("le Temps cosmique»). Les Yokut disent par exemple «le monde est passé » pour exprimer qu' "un an s'est écoulé ». M. Eliade rappelle que Hermann Usener a montré la parenté étymologique entre templum et tempus, le Temple étant le lieu sacré par excellence et l'image du Monde, sanctifiant le Cosmos tout entier. 
héritière à la fois de la première et de la deuxième Rome (Constantinople) - et l'aspect eschatologique - Moscou comme troisième mais surtout dernière Rome. La spécificité de la perception eschatologique est d'entrelacer dans un agencement équivoque l'irréversibilité du temps historique (chaque manifestation de Dieu dans l'histoire n'est pas réductible aux apparitions antérieures) avec la répétition cyclique d'événements providentiels, propre au temps cosmologique.

Mais cette compréhension du temps est indissolublement liée à une intelligence originale de l'espace: tout comme il n'y a pas d'événement historique "neutre ", qu'on ne puisse interpréter à la lumière du passé et des prophéties, il n’y a pas non plus de lieu géographique "neutre ", qui ne soit pas déterminé par une valeur singulière. Ainsi, «l'Europe» en tant qu'Autre sur le plan "spatial» (géographique) est constamment perçu comme l'hérétique, le Latin, le Suédois, l'incarnation du Mal, l'envoyé du Diable, l'Antéchist. Lorsque le jugement populaire condamne l'action d'un Tsar (les exemples les plus connus sont bien sûr Ivan le Terrible et Pierre le Grand), la sécession symbolique s'effectue au nom d'un basculement spatial (la «Sainte Terre Russe » n'est plus la terre possédée par le tsar, accusé d'être "européen » et instrument du Malin, mais elle se localise à l'opposé de l'Occident, beaucoup plus loin à l'est, là où vivent les «vrais chrétiens ", à l'instar de la mythique "ville sainte" de Kitèje) et temporel (la présence de l'Antéchrist sur le trône marque l'avant-libération, la nécessité de la rédemption collective qui sera conduite après le soulèvement contre l'usurpateur du "vrai » tsar, chargé de rétablir la coïncidence originelle entre ce dernier et son peuple). Comme on le voit, la «souveraineté universelle» qui inspire la compréhension sociale de la Russie traditionnelle ne peut pas comprendre le temps (l'histoire) et l'espace (le territoire) comme "des substances homogènes et divisible à l'infini, égales à ellesmêmes dans chacune de leurs parties " (ibid.: 306) ${ }^{14}$, ainsi que les définira l'idéologie moderne. Telle que nous l'avons cernée, la "Sainte terre russe » se révèle idée-valeur d'ouverture vers l'invisible, définissant un monde de relations qui s'oppose à la valorisation des éléments substantiels et de la complétude terrestre caractérisant la configuration territoriale moderne. Mais il faut se garder de conclusion unilatérale quant à une antinomie stricte entre Occident et Russie : le "modèle » européen ne se définit pas en effet par un passage linéaire et simple d'un mode de temporalité ou de souveraineté à un autre, mais, au contraire, par l'imbrication des deux et par la subordination progressive de l'un à l'autre. La spécificité de la Russie, y compris dirions-nous dans la période la plus contemporaine,

14. Citation légèrement modifiée, puisque l'analyse du temps linéaire est ici étendu à l'espace. 
n'est pas due à son appartenance à un mode plutôt qu'à l'autre, mais à une articulation différente des ces deux options, qui sont constitutives de toute société moderne. Saisir la complexité des diverses modalités d'instauration sociale de catégories aussi aisément objectivées que le temps et l'espace, c'est appréhender de façon directe la faculté de chaque culture à s'inscrire dans l'universel, à en révéler une facette particulière et pourtant compréhensible - sous certaines conditions - par la pratique comparative.

\section{La Russie contemporaine en perspective comparative}

Depuis l'écroulement inattendu du régime soviétique, les sciences sociales occidentales "post-kremlinologie » se sont précipitées au chevet de la Russie afin d'en publier l'avis de décès (la "part maudite " communiste) puis d'en célébrer la (re-)naissance laborieuse, par le marché et la démocratie. D’où l'utilisation rituelle d'un vocabulaire neutre en apparence, mais de facto lourd de connotations idéologiques, porteur d'explications implicites quant à l'écart évident entre la situation réelle vécue et les promesses et attentes innombrables liées au nouveau régime: "transition ", "mutation ", " développement». "L'Occident» se retrouve inévitablement édifié en modèle incarnant les valeurs "modernes", archétype de l'évolution historique vers le Progrès et le Bien-être. Évidemment, doivent demeurées inavouées sous ce discours, qu'il ne faut pas hésiter à qualifier d' " ethnocentriste", non seulement l'histoire culturelle multiséculaire qui a permis d'aboutir à la construction de ces valeurs « naturelles » et « objectives » (l'individu et la prééminence de la sphère économique), mais également les disparités d'interprétation et d'application de ces notions au sein même de l'ensemble complexe hétérogène "Occident", sans parler des conflits prégnants en terme de condition socio-économique qu'entraîne chaque jour leur réalisation effective. L'hégémonie du discours libéral individualiste se fait d'autant plus pressante qu'elle relève de plus en plus d'un impensé « idéologique » de nos sociétés, dans le sens dumontien d' «ensemble de représentations et d'idées-valeurs» (Dumont 1983), et masque par là-même la conflictualité latente et déclarée entre des positions qui se réclament toutes du même idéal (en général, sous la forme de revendications à des "droits ") mais s'opposent âprement quant à son effectuation sociale.

Si l'on veut réellement comprendre quelque chose à la situation actuelle en Russie, il convient avant tout de se déprendre des "nouveaux lieux communs " (dont l'exégèse contemporaine demanderait un travail considérable) quant à "l'État de droit » et la "démocratie ", et de renoncer à la vision catastrophiste et spectaculaire trop souvent renvoyée par les médias occidentaux (dictature, mafia, corruption), mettant en relief des pratiques 
dont personne ne conteste l'existence mais qui ne définissent pas la réalité russe dans sa globalité. Une analyse qui ne serait ni méprisante ni condescendante, et tenterait d'éviter les deux attitudes opposées mais se renforçant réciproquement, que les Russes eux-mêmes aiment parfois à entretenir: d'une part, l'isolationnisme du "seul contre tous", la grande puissance incomprise et, d'autre part, l'auto-flagellation, l'humiliation permanente devant la décomposition du lustre d'antan.

Il est rassurant de noter une tendance croissante à la complexification dans les études consacrées à la situation en Russie, comme en témoignent les dossiers " est-européens » des revues Mouvements ${ }^{15}$ ou Le Débat ${ }^{16}$, expliquant notamment "pourquoi la construction d'un régime démocratique n'est pas la priorité des Russes" (Mendras 1999: 36). Marie Mendras insiste sur «le décalage entre la construction institutionnelle et politique et les réalités de la vie quotidienne, la forte emprise des administrations locales et régionales, un rapport aux droits et aux institutions qui s'est fort peu "européanisé" et qui révèle toujours une préférence pour le flou et l'interprétation ». L'observation selon laquelle ce « règne du flou et de l'interprétation ne résulte pas d'une dérive mais bien d'une préférence» (ibid.: 43) renvoie à la nécessité de replacer la situation contemporaine dans une perspective historique et culturelle longue, apte à expliciter les divers niveaux significatifs relevés. Ainsi, la "préférence pour le flou "s'exprime par une propension à traiter les règles juridiques comme des normes conjoncturelles, susceptibles d'interprétations variables, par la prépondérance d'un système de dépendances (notamment bureaucratiques) complexe mais caractérisé (à l'encontre des analyses wébériennes classiques décrivant le pouvoir administratif « légal-rationnel » moderne comme processus d'abstraction de la règle) par une "personnalisation " exacerbée ${ }^{17}$, ou encore par l'inexistence de l'État "au sens démocratique de représentant et garant de la chose publique ${ }^{18}$. Or, ces spécificités de la Russie

15. Mouvements, 1999, $\mathrm{n}^{\circ} 6:$ Est 89-99: Que sont les espoirs devenus?, avec quelques remarquables contributions remettant en cause le modèle hégémonique de "transition ", voir Pierre Rolle, "Y a-t-il une fin à la transition?" (pp. 31-40) ou encore Jacques Sapir, "Réflexions théoriques à partir de la transition : une crise des paradigmes?" (pp. 52-62).

16. Le Débat, nov.-déc. $1999, \mathrm{n}^{\circ} 107$, avec, outre l'article de Marie Mendras cité infra, les contributions importantes de Georges Nivat et Anatoli Vichnievski (dont le dernier ouvrage traduit, La Faucille et le rouble, est recensé par Krzysztof Pomian).

17. Cette "personnalisation" des rapports peut sans doute se comprendre comme forme locale de "clientélisme", mot passe-partout qui désigne en fait la perpétuation, chaque fois particulière, de relations sociales à travers l'importation des règles juridiques de l'État de droit à l'occidentale, impersonnelles, universelles et abstraites: la retraduction de ces dernières dans une hiérarchie de valeurs non totalement imprégnée d'individualisme aboutit ainsi à la compréhension des solidarités et appartenances autochtones (qu'elles soient familiales, locales, religieuses ou autres) dans un vocabulaire essentiellement à connotation péjorative, comme " clientélisme " ou « corporatisme ». 
actuelle, partiellement incompréhensibles si l'on se réfere seulement aux processus de "démocratisation ", reçoivent un éclairage particulier sous les lumières de la comparaison socio-anthropologique, par exemple à partir des études sémiotiques de Youri Lotman et Boris Ouspenski, qui, depuis de nombreuses années, se sont attachés à décrire «le caractère dualiste de la culture russe » marqué par l'absence de "zone intermédiaire», neutre et objective, au sein de laquelle peut se développer la notion même d' «État de droit ${ }^{19}$. Il existe aujourd'hui, en Russie même, des œuvres entières encore inexploitées, peu ou pas traduite, dans le domaine des sciences sociales, œuvres assises sur un comparativisme rigoureux avec les données occidentales, et dont la lecture serait pour nous, dans un juste retour des choses, source d'un enrichissement considérable pour l'appréciation contemporaine d'une dynamique historique à bien des égards incertaine et contradictoire ${ }^{20}$.

MOTS-CLÉS/KEYWORDS: Russie/Russia - tsar - souveraineté/sovereignty - temps/time espace/space.

18. «Encore aujourd'hui, le Russe se considère vivre hors du champ étatique et n'imagine pas avoir un intérêt à participer à la vie publique. Il n'imagine pas non plus que l'État a pour fonction première l'intérêt de la société. Les deux mondes - l'État et la société - restent séparés, même si quelques passerelles ont été installées grâce aux libertés, aux médias et à la nécessité pour les élites de "faire de la politique" afin d'accommoder l'institution électorale" (Mendras 1999: 46). Cette analyse est corroborée par les études sociologiques montrant le degré de "refus de la politique", y compris chez certains militants démocratiques "par obligation", dont l'action est comprise par eux-mêmes comme éphémère et circonstancielle : l'État et l'élite politique censée lui donner les orientations principales à partir de la volonté du peuple (exprimée dans le vote) sont considérées comme profondément séparés de la "société ». Voir Berelowitch \& Wieviorka (1996 : 92), avec des citations significatives de militants politiques : "J'espère pouvoir un jour m'occuper de mon travail et laisser la politique aux professionnels à qui je fais confiance..." , ou "Il est peu rationnel que nous, des non-professionnels, nous occupions de politique ». En 1997, plus de la moitié des personnes interrogées (52\%) répondent à la question : "Qui selon vous dirige la Russie?", par "la mafia, le crime organisé ", ce qui en dit long sur le discrédit frappant les gouvernants russes (sondage publié dans le Moskovskij Komsomolets, 5 sept. 1997).

19. Lotman \& Ouspenski 1990, avec notamment les textes « La dualité des modèles et son rôle dans la dynamique de la culture russe" (21-56) et "Deux modèles archétypes de culture : "conclure un pacte" et "s'en remettre à autrui"» (140-155). Cette approche est notamment reprise et synthétisée dans le dernier ouvrage de Y. Lotman, publié en Russie post-communiste, faisant de l'accès à une "structure trinaire " par les liens développés avec l'Occident, la seule possibilité "démocratique " de la rupture : Lotman 1992. 20. Par exemple, D. S. Likhatchov, A. M. Pantchenko, V. M. Jivov, V. N. Toporov, B. F. Egorov, etc. À souligner l'important et érudit travail de Françoise Lesourd, qui a traduit Likhatchov (1988) et commenté son travail (Lesourd 1988). 
Beaud, Olivier

1996 "Souveraineté », in Philippe Raynaud \& Stéphane Rials, eds, Dictionnaire de philosophie politique. Paris, PUF : 625-633.

\section{Berelowitch, Alexis \& Michel Wieviorka}

1996 Les Russes d'en bas: enquête sur la Russie post-communiste. Paris, Le Seuil.

\section{Besançon, Alain}

1967 Le Tsarévitch immolé. Paris, Plon.

\section{Boureau, Alain \& Claudio Ingerflom, eds}

1992 La Royauté sacrée dans le monde chrétien. Colloque de Royaumont, mars 1989. Paris, Éditions de l'EHESS.

\section{Castoriadis, Cornelius}

1975 L'Institution imaginaire de la société. Paris, Le Seuil.

\section{Christoff, Peter K.}

1961 Introduction to Nineteenth-Century Russian Slavophilism : A Study in Ideas.

Vol. I. 's-Gravenhage, Mouton.

1972 Introduction to Nineteenth-Century Russian Slavophilism : A Study in Ideas.

Vol. II. The Hague, Mouton.

\section{Coppet, Daniel de}

1998 «Une monnaie pour une communauté mélanésienne comparée à la nôtre pour l'individu des sociétés européennes ", in Michel Aglietta \& André Orléan, eds, La Monnaie souveraine. Paris, Odile Jacob: 159-211.

\section{Dagron, Gilbert}

1996 Empereur et prêtre. Études sur le "césaropapisme " byzantin. Paris, Éditions du Cerf.

\section{Dennes, Maryse}

1987 Le Baptême de la Russie: mille ans de foi chrétienne. Paris, Nouvelle Cité.
Dumont, Louis

1983 Essais sur l'individualisme:

une perspective anthropologique sur l'idéologie moderne. Paris, Le Seuil.

Eliade, Mircea

1965 Le Sacré et le profane. Paris, Gallimard.

\section{Evdokimov, Michel}

1987 Pèlerins russes et vagabonds mystiques. Paris, Éditions du Cerf.

\section{Gauchet, Marcel}

1977 «La dette du sens et les racines de l'État: politique de la religion primitive", Libre 2. Paris, Payot: 5-43. 1981 «Des deux corps du roi au pouvoir sans corps. Christianisme et politique. Première partie ", Le Débat 14 : 133-157.

1985 Le Désenchantement du monde: une histoire politique de la religion. Paris, Gallimard.

\section{Goldfrank, David M.}

1984 «L'utopisme dans la Russie d'Ivan le Terrible: une interprétation de la pensée russe au XVI ${ }^{\mathrm{e}}$ siècle", Revue des Études slaves 56 (4) : 591-598.

\section{Gratieux, André}

1939 A. S. Khomiakov et le mouvement slavophile. Paris, Éditions du Cerf, 2 vol.

\section{Heller, Leonid \& Michel Niqueux}

1995 Histoire de l'utopie en Russie. Paris, PUF.

Ingerflom, Claudio Sergio

1992 «Les représentations collectives du pouvoir et "l'imposture" dans la Russie des XVIII'-XIXe siècles ", in Alain Boureau \& Claudio Sergio Ingerflom, eds, La Royauté sacrée dans le monde chrétien... : 157-164. 1993 "Oublier l'État pour comprendre la Russie? (XVI ${ }^{\mathrm{e}}$-XIX ${ }^{\mathrm{e}}$ siècles) : excursion historiographique ", Revue des Études slaves 66 (1) : 125-134. 
1996 «Entre le mythe et la parole : l'action. Naissance de la conception politique du

757.

\section{Laran, Michel \& Jean Saussay}

1975 La Russie ancienne (IX'-XVII siècles). Paris, Masson.

\section{Lesourd, Françoise}

1988 «Dmitri Likhatchov, historien et théoricien de la littérature ", in D. Likhatchov, Poétique historique de la littérature russe. Lausanne, L'Âge d'Homme : 255-321.

\section{Likhatchov, Dmitri}

1988 Poétique historique de la littérature russe du X au XX siècle. Lausanne, L'Âge d'Homme.

\section{Lotman, Youri}

1992 Culture et explosion (Koultoura i vzryv). Moscou, Progress.

\section{Lotman, Youri \& Boris Ouspenski}

1990 Sémiotique de la culture russe. Lausanne, L'Âge d'Homme.

\section{Maine, Henri Sumner}

1861 Ancient Law. Its Connection with the Early History of Society, and its Relation to Modern Ideas. Oxford, Oxford University Press.

\section{Mendras, Marie}

1997 "Le mythe du nationalisme russe", in Pierre Birnbaum, ed., Sociologie des nationalismes. Paris, PUF : 85-102.

1999 «La préférence pour le flou : pourquoi la construction d'un régime démocratique n'est pas la priorité des Russes ", Le Débat 107 : 35-50

Meyendorff, Jean

1959 Saint Grégoire Palamas et la mystique orthodoxe. Paris, Le Seuil.

\section{Ouspensky, Leonide}

1982 La Théologie de l'icône dans l'Église orthodoxe. Paris, Éditions du Cerf.

\section{Poliakov, Léon}

1989 Moscou troisième Rome: les intermittences de la mémoire historique. Paris, Hachette.

\section{Raeff, Marc}

1982 Comprendre l'Ancien Régime russe. Paris, Le Seuil.

Raynaud, Philippe \& Stéphane Rials, eds 1996 Dictionnaire de philosophie politique. Paris, PUF.

\section{Rouleau, François}

1990 Ivan Kireievski et la naissance du slavophilisme. Namur, Culture et vérité.

\section{Schaub, Marie-Karine}

1992 «Les couronnements des tsars en Russie du XVI ${ }^{\mathrm{e}}$ au XVIII ${ }^{\mathrm{e}}$ siècle. Essai d'historiographie", in Alain Boureau \& Claudio Sergio Ingerflom, eds, La Royauté sacrée dans le monde chrétien... : 139-148.

\section{Siniavski, André}

1990 Ivan le Simple: paganisme, magie et religion du peuple russe. Paris, Albin Michel.

\section{Spidlik, Tomas}

1994 L'Idée russe: une autre vision de l'homme. Troyes, Fatès.

\section{Strada, Vittorio}

1992 «Fin d'un empire, naissance d'une nation ", in Marie Mendras, ed., Un État pour la Russie. Bruxelles, Complexe.

\section{Szamuely, Tibor}

1976 La Tradition russe. Paris, Stock.

\section{Tchistov, Kirill V.}

1996 "L'utopie sociale en Russie (à travers les légendes populaires et l'expérience contemporaine ", Ethnologie française 26 (4) : Russie: par oles russes: 628-640. 
Vibert, Stéphane

1999 La Quête russe de l'Universel: mouvement slavophile et hiérarchie de valeurs sociocommunautaire (1825-1855). Paris, École des hautes études en sciences sociales, thèse de doctorat.

Vodoff, Vladimir

1988 Naissance de la chrétienté russe. Paris, Fayard.
Walicki, Andrej

1980 The Slavophile Controversy. History of a Conservative Utopia in Nineteenth-

Century Russian Thought. Oxford,

Clarendon Press.

Zelenin, Dmitri K.

1994 Izbrannyie troudy - Stati po doukhovnoi koultoure (Travaux choisis). Moscou, Indrik.

\section{RÉSUMÉ/ABSTRACT}

Stéphane Vibert, La Russie, le temps et l'espace. Transformations du socio-cosmisme et construction d'une modernité hybride. - L'évolution de la compréhension sociale des catégories de temps et d'espace dans la Russie tsariste puis impériale (XVI ${ }^{\mathrm{e}}$-XVIII ${ }^{\mathrm{e}}$ siècles) apporte un éclairage sur la dynamique propre à la "matrice théologico-politique" russe dans une perspective comparative. La reconduction d'une souveraineté universelle "socio-cosmique " s'effectue au travers d'un changement d'instance incarnatrice. Jadis dévolu au Tsar comme "dieu terrestre", le messianisme eschatologique s'inscrit au début XVIII ${ }^{e}$ siècle dans le peuple théophore vivant sur la «Sainte Terre russe », créant les conditions d'une "modernité politique» originale et problématique.
Stéphane Vibert, Russia, Time and Space. Transformations of the socio-cosmism and Construction of an Hybrid Modernity. - The evolution in the social understanding of the categories of time and space in Czarist Russia (16th-18th centuries) places the momentum of the Russian "theologico-political matrix " in a comparative perspective. A universal "socio-cosmic sovereignty" was continued by changing the level incarnating it. Formerly attributed to the Czar as a «terrestrial god", eschatological messianism inhered, in the early 18th century, in a "theophoric people" living on "holy Russian soil", thus creating the conditions for an original and problematical political modernity. 